\begin{tabular}{|c|}
\hline $\begin{array}{c}\text { PRAMANA } \\
\begin{array}{c}\text { journal of } \\
\text { physics }\end{array}\end{array}$ \\
\hline
\end{tabular}

\title{
Development of a microstrip-based neutron detector
}

\author{
S S DESAI $^{1}$, A M SHAIKH $^{1}$, V RADHAKRISHNA ${ }^{2}$ and K RAJANNA ${ }^{2}$ \\ ${ }^{1}$ Solid State Physics Division, Bhabha Atomic Research Centre, Mumbai 400 085, India \\ ${ }^{2}$ Department of Instrumentation, Indian Institute of Science, Bangalore 560 012, India \\ E-mail: shaikham@magnum.barc.ernet.in
}

\begin{abstract}
A gas-filled microstrip detector for thermal neutrons has been built and successfully tested in our laboratory. The detector has an active area of $20 \mathrm{~mm} \times 15 \mathrm{~mm}$ and consists of alternate anodes and cathodes of widths $12 \mu \mathrm{m}$ and $300 \mu \mathrm{m}$ respectively. The anode to cathode gap is $150 \mu \mathrm{m}$ and the pitch is $612 \mu \mathrm{m}$. A high resistance, meandering type horizontal strip connects the anodes at one end and aids in position sensing by charge division method. The detector is tested with gas mixtures ${ }^{3} \mathrm{He}+\mathrm{Kr}(1: 2)$ and ${ }^{3} \mathrm{He}+\mathrm{CF}_{4}(2: 1)$ at pressure of 3 atmospheres and using a $\mathrm{Pu}$-Be neutron source. The pulse height spectrum shows energy resolution of $\sim 8 \%$ (FWHM) for the $764 \mathrm{keV}$ peak at anode voltage of $525 \mathrm{~V}$ for ${ }^{3} \mathrm{He}+\mathrm{Kr}$ and $\sim 15 \%$ at anode voltage of $800 \mathrm{~V}$ for ${ }^{3} \mathrm{He}+\mathrm{CF}_{4}$. Gas gains up to $6.3 \times 10^{3}$ and $3.6 \times 10^{3}$ are obtained respectively with these gas mixtures. The overall efficiency of the detector along the sensitive length is tested by exposing the active area to neutrons and recording the position spectrum. The detector shows fairly uniform efficiency $(\sim 45 \%)$ over the active length.
\end{abstract}

Keywords. Microstrip gas counters; gas-filled proportional counters; position-sensitive detectors; neutron scattering and diffraction.

PACS Nos 29.40.-n; 29.40.Cs; 29.40.Gx; 61.12.-q

\section{Introduction}

Microstrip gas counters (MSGC) are of great interest for researchers around the world since their introduction by Anton Oed in 1988 [1]. They can be used as position sensitive detectors for X-rays and neutrons [2-6] and are superior to conventional multiwire proportional counters (MWPC) in terms of resolution and count rate. The performance of the detector can be tailored using advanced thin film technology and lithography. As part of our detector development programme we have developed a MSGC in collaboration with Indian Institute of Science, Bangalore. The fabricated MSGC has been tested for its performance with both X-rays and neutrons. The details of the fabrication and X-ray test results have been reported elsewhere $[7,8]$. We report here preliminary results obtained on performance of MSGC namely, energy resolution, gain characteristics, efficiency and position resolution with thermal neutrons. 

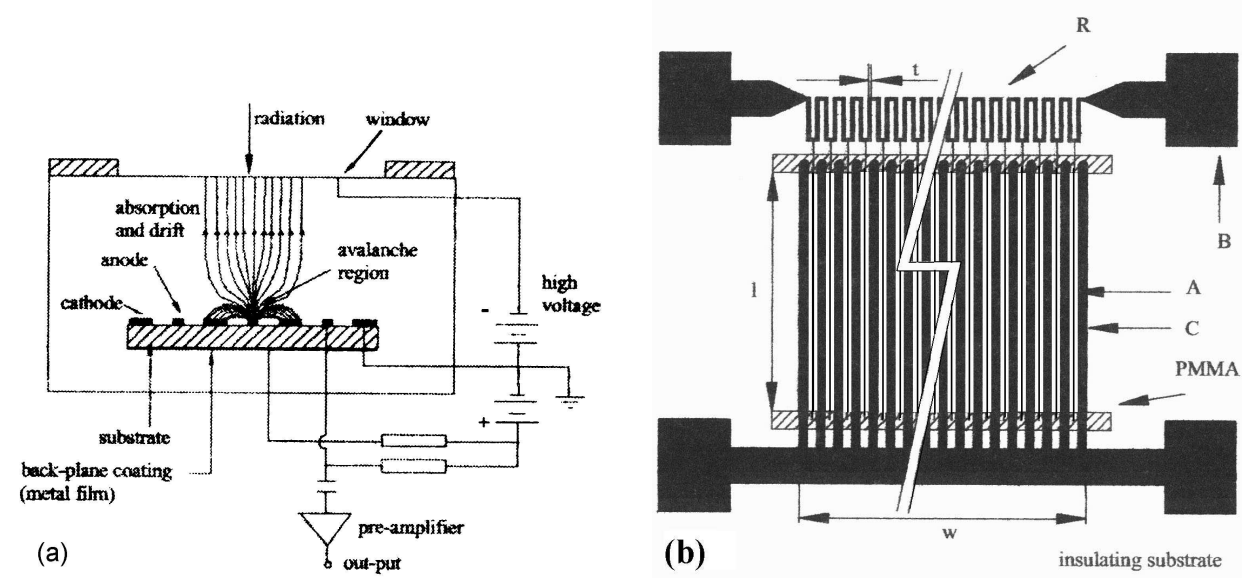

Figure 1. (a) Schematic of the cross-sectional view of MSGC. The anode and cathode strips shown normal to the plane of the paper. (b) Schematic of the designed microstrip pattern on insulating substrate. A, anode; C, cathode; $\mathrm{B}$, bonding pad; $\mathrm{R}$, meandering resistive strip; $\mathrm{t}$, meandering strip width, PMMA, polymethyl methacrylate coating for end passivation; w, active area width; l, active area length.

\section{Microstrip gas chamber}

A cross-sectional view of a microstrip detector is shown in figure 1a. The most important aspect of microstrip design is that the closely spaced anode and cathode wire planes of the MWPC are replaced by alternating anode and cathode strips of a few microns width, deposited in a single plane on a suitable insulating substrate. The applied electric potential alternates between each strip. An electron produced anywhere in the gas volume due to primary ionization travels along the field lines (in drift region) towards the anode and enters the region of high electric field near the anode strips (avalanche region) where it will gain sufficient energy. This energetic electron will produce an avalanche of electrons in the gas medium due to secondary ionization. The formation of electron avalanche results in gas multiplication or gas gain. The secondary electrons move to anodes and the ions drift towards nearby cathodes giving rise to signals on the anode and cathode strips, which are then routed through corresponding electronic systems. As per the scheme, each electrode needs its own electronic detection system. This can however, be simplified by the incorporation of a resistive chain along the anodes. The signals can be amplified by external amplifiers and analysed.

A typical microstrip plate designed is shown schematically in figure $1 \mathrm{~b}$ and design parameters are given in table 1. A horizontal strip connects the anodes at one end and has high resistance per unit length compared to that of the anode and cathode strips. High resistance thus provided between any two anode strips aids in position sensing by charge division method. A meandering resistive structure is adapted in order to increase the position sensitivity. Chromium is chosen as material for all the conducting strips because it has high conductivity, good chemical stability, good 
Table 1. Design parameters of microstrip.

\begin{tabular}{ll}
\hline Substrate & Borosilicate glass \\
Anode width & $12 \mu \mathrm{m}$ \\
Cathode width & $300 \mu \mathrm{m}$ \\
Anode-cathode gap & $150 \mu \mathrm{m}$ \\
Pitch & $612 \mu \mathrm{m}$ \\
Active area & $20 \mathrm{~mm} \times 15 \mathrm{~mm}$ \\
Resistive strip width & $100 \mu \mathrm{m}$ \\
Resistive strip resistance & $44 \mathrm{k} \Omega$ \\
\hline
\end{tabular}

adhesion to the glass substrate and withstands mild discharges due to its high melting point. DC magnetron sputtering is used for depositing the film and the required pattern for the conducting strips is obtained using photolithography technique. The lift off lithography method is used to generate bonding pads. Borosilicate glass with surface resistivity of the order of $\sim 10^{15} \Omega /$ sq is used as substrate.

\section{Detector performance}

The detector is tested for its performance with various gas mixtures, such as ${ }^{3} \mathrm{He}+\mathrm{Kr}(1: 2)$ and ${ }^{3} \mathrm{He}+\mathrm{CF}_{4}(2: 1)$ filled at a pressure of 3 atm and using a $\mathrm{Pu}-$ Be neutron source. $\mathrm{Kr}$ or $\mathrm{CF}_{4}$ is added as the stopping and quenching gas. The cathodes are maintained at zero potential. A drift gap of $16 \mathrm{~mm}$ is maintaned throughout the experiment. The anode voltage is adjusted to obtain output response from the detector. A charge sensitive pre-amplifier is connected to one end of the resistive strip. Linear amplifier is used for shaping the signal pulse from the pre-amplifier. The output pulse from the linear amplifier is observed on the oscilloscope screen and also fed to a PC-based multichannel analyser (PC-MCA) and the pulse height spectrum is recorded. The pulse height spectrum recorded

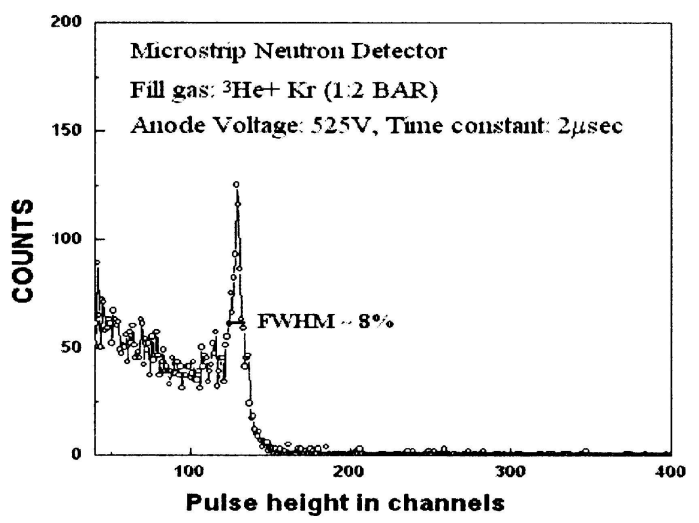

Figure 2. Pulse height spectrum measured with MSGC using Pu-Be neutron source. 


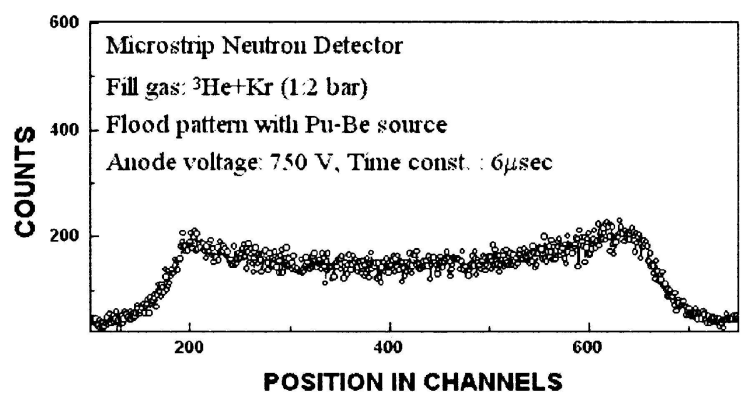

Figure 3. Overall efficiency of the detector along the sensitive length, with neutrons from $\mathrm{Pu}-\mathrm{Be}$ source.

for ${ }^{3} \mathrm{He}+\mathrm{Kr}$ gas mixture is shown in figure 2. The peak corresponding to $764 \mathrm{keV}$ energy released in ${ }^{3} \mathrm{He}(\mathrm{n}, \mathrm{p})$ reaction is well-resolved and discriminated from that of accompanying gammas in both the cases. The contribution due to wall effect is also seen in the pulse height spectrum.

From the pulse height spectrum, one can estimate various detector parameters such as its energy resolution, energy linearity, gas gain etc. Their dependence on the operating conditions like anode voltage, gas composition and pressure, drift gap etc. can also be studied. As seen in figure 2, an energy resolution of $8 \%$ (FWHM) is obtained. The gas gain of the detector as function of anode voltage is calculated by the procedure given elsewhere [8], and a gain of $6.3 \times 10^{3}$ and $3.6 \times 10^{3}$ is obtained for the ${ }^{3} \mathrm{He}+\mathrm{Kr}$ and ${ }^{3} \mathrm{He}+\mathrm{CF}_{4}$ gas mixtures respectively.

The overall efficiency of the detector along the sensitive length is tested by exposing the active area to neutrons from a $\mathrm{Pu}-\mathrm{Be}$ source and recording the position spectrum using charge division method [8]. The detector shows fairly uniform efficiency $(\sim 35-55 \%)$ over the active length (figure 3 ).

Position resolution of the detector is determined by measuring detector response (figure 4) to a finely collimated, $1.5 \mathrm{~mm}$ wide beam of neutrons from Apsara reactor, incident normally on the window. The position spectrum on interpolation indicates, intensity spread along three consecutive strips due to beam width and size of the charge centroid. Even though central strip acquiring maximum intensity shows a spread of 12 channels (FWHM), taking into account broadening in the base region, position resolution is estimated to around three times the pitch of the detector $(\sim 1.8 \mathrm{~mm})$. It can be further improved by increasing the gas pressure.

\section{Conclusion}

A gas-filled microstrip detector with an active area of $20 \mathrm{~mm} \times 15 \mathrm{~mm}$ has been developed and its performance tested with thermal neutrons. The preliminary results on energy resolution, gas gain, efficiency and position resolution are comparable to characteristics of similar detectors developed in various laboratories abroad [2-6]. The present results are obtained with a drift gap of $16 \mathrm{~mm}$, i.e. the sensitive volume of the detector is restricted to only $\sim 5$ cc resulting in low sensitivity and count rate. Further testing of the detector with higher drift gap, drift voltage and gas pressure 


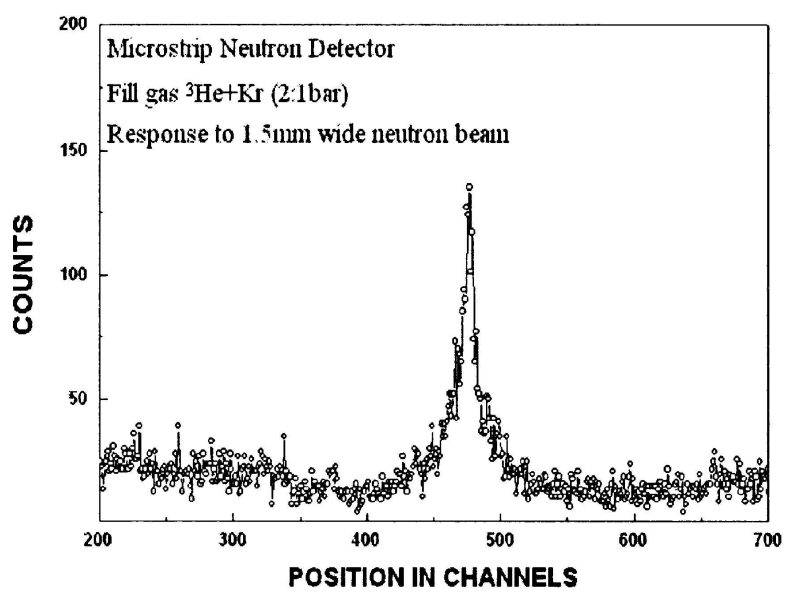

Figure 4. Position response of $1.5 \mathrm{~mm}$ wide, $4 \AA$ neutron beam.

is in progress. Various parameters controlling the total performance of the detector for neutron detection are still to be optimized. The present microstrip detector is intended to use as a module for making banana-type neutron detector.

\section{Acknowledgement}

The work reported in this paper has been supported by the Board of Research in Nuclear Sciences (BRNS), Department of Atomic Energy, India through a research project. The authors acknowledge Dr K Natrajan, Bharat Electronics Ltd., Bangalore for his help in getting microstrip plates fabricated.

\section{References}

[1] A Oed, Nucl. Instrum. Methods A263, 351 (1988)

[2] A Oed, P Convert, M Berneron, H Junk, C Budtz-Jorjensen, M M Madsen, P Jonasson and H W Schnopper, Nucl. Instrum. Methods A284, 223 (1989)

[3] B Gebauer, Ch Schulz, Th Wilpert and S F Biagi, Nucl. Instrum. Methods A409, 56 (1998)

[4] J E Bateman, J F Connolly, G E Derbyshire, D M Duxbury, J Lipp, J A Mir, R Stephenson, J E Simmons and E J Spill, RAL-TR-1999-056

[5] J E Bateman, N J Rhodes and R Stephenson, Nucl. Instrum. Methods A477, 365 (2002)

[6] J D Lipp, J E Bateman, G E Derbyshire, I W Kirkman, G van der Laan, R Stephenson and C M Teodorescu, J. Synchrotron Rad. 10, 455 (2003)

[7] V Radhakrishna, Ph.D. Thesis (Indian Institute of Science, Bangalore, 2002)

[8] V Radhakrishna, S S Desai, A M Shaikh and K Rajanna, Curr. Sci. 84(10), 1327 (2003) 